\title{
Cluster Head Selection and Routing Protocol for Wireless Sensor Networks (WSNs) Based on Software-Defined Network (SDN) Via Game of Theory
}

\author{
Seyed Salar Sefati ${ }^{1, ~ *}$, Sara Ghiasi Tabrizi ${ }^{2}$ \\ ${ }^{1}$ Department of Computer Engineering, Institute of Higher Education Roshdiyeh, Tabriz, Iran \\ ${ }^{2}$ Department of Computer Engineering, Tabriz Branch, Islamic Azad University, Tabriz, Iran
}

Email address:

Sefati@ roshdiyeh.ac.ir (S. S. Sefati)

*Corresponding author

To cite this article:

Seyed Salar Sefati, Sara Ghiasi Tabrizi. Cluster Head Selection and Routing Protocol for Wireless Sensor Networks (WSNs) Based on Software-Defined Network (SDN) Via Game of Theory. Journal of Electrical and Electronic Engineering. Vol. 9, No. 4, 2021 , pp. $100-115$. doi: $10.11648 /$ j.jeee.20210904.12

Received: May 31, 2021; Accepted: July 6, 2021; Published: July 23, 2021

\begin{abstract}
There is a way to prolong the life of sensor networks according to which a hierarchical routing algorithm is used intelligently, which employs all network elements in data transmission. Clustering the nodes is one of the best methods that can significantly increase the network life. Making a cluster, selecting a Cluster Head $(\mathrm{CH})$ and data transmission in Wireless Sensor Network (WSN) are the issues that affect energy consumption. Software-Defined Networks (SDN) are a modern network architecture that distinguishes the network control panel from the data plate also this architecture cause the network utilizing is increased, and the operational cost is reduced. This method also causes creativity and perfection in the network area. Moreover, the possibility of implementing management protocols, including traffic management, which is an inevitable part of networks, can be implemented in SDN with a higher level of flexibility. In this paper, the $\mathrm{CH}$ has selected trough game theory, which sends data with the help of game theory rewards and calculating the geographical location of other nodes. Then, high-priority data is sent according to the proposed algorithm with the help of game theory. The simulation results in NS3 software show that the proposed method has obtained acceptable results compared with Artificial Bee Colony algorithm (ABC), Genetic Algorithm (GA), Cuckoo Search algorithm (CS), Firefly algorithm (FA) and Grey Wolf Optimization Algorithm (GWO).
\end{abstract}

Keywords: Wireless Sensor Network, Software Defined Network, Routing, Cluster Head Selection, Game Theory

\section{Introduction}

The Software Defined Network (SDN) is an emerging network architecture that allows overcoming network infrastructure in current limitations [1]. This means that an intelligent controller configures transmission elements with sending rules for different packets. SDN is the next transformation in computer networks [2]. When a packet reaches a switch in SDN, a software in the switch decides where the packet should be sent [3]. SDN are a centralized program for implementing the network and monitors the network behavior [4]. The main purpose is to separate the control panel from the data plate. This separation can be done by the Application Programming Interface (API) between the switches and the controller [5]. There are advanced network policies such as load balancing, routing and implementable security [6]. Each network must be configured based on the requirements and efficiency objectives. Network reliable configuration it depends on meeting performance, security, and policies [7].

Small sensors called wireless sensor networks (WSN) that can receive various environmental information based on the type of sensor and processing [8]. WSN consists of many nodes that are widely distributed in an environment and collect information from the environment [9]. The location of the nodes is not necessarily predetermined and unknown [10]. This feature allows leaving them in dangerous or inaccessible places. WSN uses various nodes to measure 
several parameters in the field of activity and enables monitoring accuracy and sending information remotely [11]. These systems can dramatically contribute the communication with the least delay high availability and that can help reduce maintenance costs [12]. It is not possible to charge the batteries in WSNs because the nodes are often in special conditions [13].

The network topology in the WSN is generally not fixed because new nodes are added and old nodes die, causing the network topology to change frequently. The SDN controller can be used in the SDN-WSN environment to obtain a dynamic network and ensure coverage. A routing protocol [14] is primarily responsible for transferring data packets between nodes. The SDN controller in the SDN-WSN network is used to send data to nodes based on a predefined route selection. The SDN-WSN controller can manage nodes and networks in an integrated Network Management System (NMS) [15]. Specific features of SDN-WSN easily add and remove nodes. Moreover, the controller in SDN-WSN can operate between the sink and the cluster head $(\mathrm{CH})$. In contrast, the sink is only responsible for receiving information or acts as cloud computing.

In the WSN, transmits data between the base station and nodes is called routing [16]. On the one hand, routing can be considered as a method of data transfer between the nodes. On the other hand, data transfer between nodes and the sink can be defined as routing [17], which are arranged to cover a large geographical area, however in the most of the time wireless sensors are mobile and move away from the base station [18]. This method of data transmission leads to saving energy and a significant increase in telecommunication between nodes and base station [19]. In the network's hierarchical structure, each node has a leader called the $\mathrm{CH}$ and $\mathrm{CH}$ usually performs specific tasks [20]. In addition, there are a number of common nodes in each cluster as members of that cluster [21]. The clustering process creates a two-level hierarchy, the upper level of which is made up of $\mathrm{CH}$ nodes and the lower level of which consists of normal nodes. $\mathrm{CH}$ nodes collect data and send it to the base station [18]. Sending to the base station can be done directly or in the form of connection with other CHs [22].

In this paper, the management of nodes and $\mathrm{CH}$ is considered with the SDN. This controller is located between the sink and the $\mathrm{CH}$, which manages the network. In this article, first $\mathrm{CH}$ is selected by game theory. If a $\mathrm{CH}$ selected in the first round, a reward is considered, then check the normal nodes have been connected with $\mathrm{CH}$ or not. $\mathrm{CH}$ duty is a heavy task in the network [23], it must be able to send all the data to the controller and have the high throughput and energy. Then, the game's score begins to be calculated; if the score has a high rate, the $\mathrm{CH}$ node is definitely selected as the $\mathrm{CH}$. In this article, after discovering the $\mathrm{CH}$, the controller commands the other nodes to route and send their own data to $\mathrm{CH}$. In this method, game theory is used only in high priority data, which is done by the First in First out (FIFO) priority queue. Low priority data is done by estimating node factors with the received power signal.
Finally, routing takes place. The main features of this paper are as follows:

Achieving the best $\mathrm{CH}$ for WSN in the area based on game theory criteria.

Selecting the efficient $\mathrm{CH}$ based on game theory for WSN.

Increasing the network lifetime, throughput, and delivery rate using game theory.

Fast routing for high priority data with the help of game theory.

The rest of the research is as follows:

Section 2 presents related work. Section 3 provides the proposed algorithm. Section 4 shows the experimental and simulation results. Finally, section 5 presents future discussions and studies.

\section{Related Works}

Numerous types of research about $\mathrm{CH}$ selection and routing protocol in WSN have been performed. This part refers to certain papers investigating the $\mathrm{CH}$ selection and routing to feature their fundamental points of interest and impediments. In addition, according to the importance of $\mathrm{CH}$ selection and routing efficiency, the QoS factors have been considered in analyzing the previous techniques. These two aspects are discussed in the following. There are several different classifications of clustering algorithms based on the characteristics and utility of the sensors within the clusters. As a consequence, it could be defined using cluster head determination, calculation complexity, or cluster arrangement criteria figure 1 shows the $\mathrm{CH}$ selection classification.

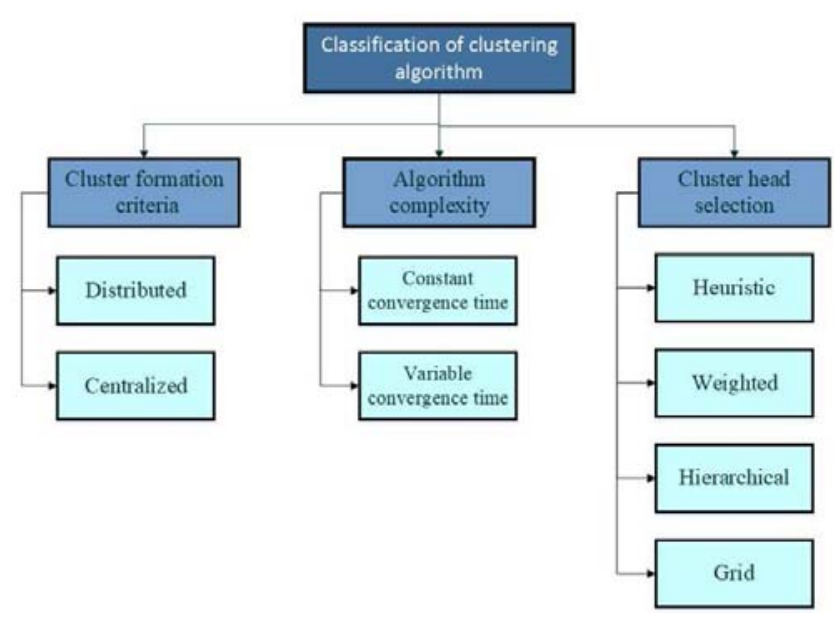

Figure 1. Classification of Clustering Algorithms.

The WSN routing protocols are classified dependent on their method of service, network configuration, and sensor node participation types. Protocols for mode of operation may be constructive, reactive, or hybrid. The protocols in participation mode could be flat, direct, or clustering dependent. Protocols may be data-centric, locationdependent, hierarchical, or QoS (Quality of Service) based in network structure mode. Figure 2 shows the classification of routing protocol in WSN. 


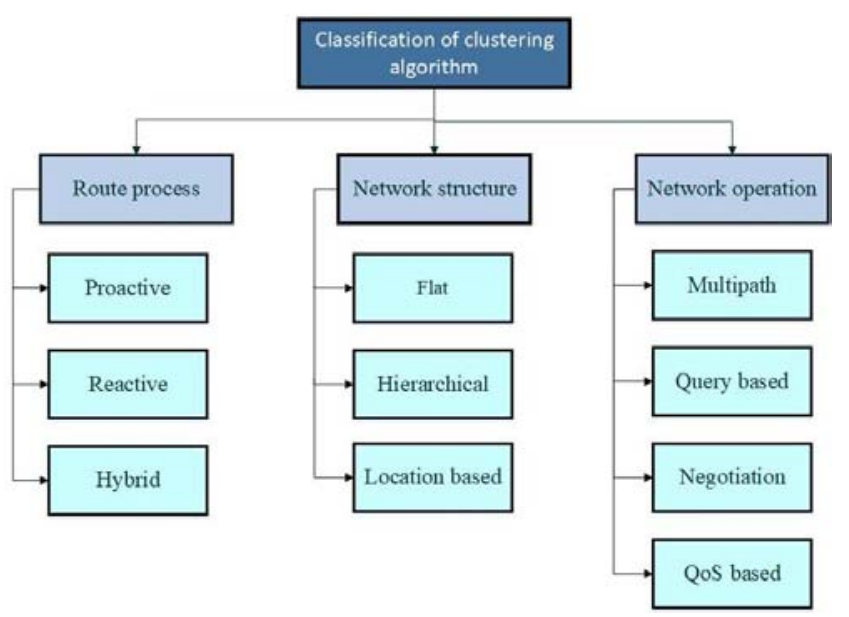

Figure 2. Classification of routing protocol.

Deepa and Latha [24] introduced a routing algorithm called Hybrid Hierarchical Secure Routing Protocol (HHSRP). The proposed algorithm is an improved approach by adding the $\mathrm{CH}$ in the network and calculating packet transmission efficiency based on packet priority. Therefore, the package can be quickly and easily delivered to the destination. The proposed approach can transmit the packet to the destination without losing the packet to any malicious node and inactive node activity. Identifying packet priority and transmitting packet to the destination is done by generating route, that is directly related to the shortest route and the head-coordinating node. The assessment outcomes indicated that the mentioned method could increase reliability and average number of packets received. Nevertheless, the mentioned technique has low load balancing and low mobility.

Sharma, et al. [25] provided a routing algorithm for WSNs. The algorithm includes clustering in WSN called Efficient Routing Algorithm (ERA) routing steps. The propose method, a novel routing scheme that provides increased throughput with managed delays while remaining power conscious. ERA is an adaptive routing algorithm that prevents congestion and helps to reduce network hotspots. They have shown that there is no need to exchange control messages to select the $\mathrm{CH}$. They have also developed an efficient strategy for organizing all nodes for different levels to build a conducted virtual column to facilitate data routing to the sink. They also devised a simple method for ensuring that all nodes participate in the data routing process. Their energy is measured at the same time based on their residual energy. Each sensor appear that the proposed protocol has message complexity and time complexity for WSNs. This method has a good performance in throughput and energy consumption. However, it has limited functionality in reliability and service monitoring.

Deebak and Al-Turjman [26] provided a routing and energy-efficient routing protocol in WSN to reduce node temperature as well as reduce critical data delay using polymorphic (not uniform) sensor nodes and called the Authentication and Encryption Model (ATE). Base stations in the network architecture of this project is in the center, but the nodes with high data rates are located in places with less mobility. In query-based or critical data packets, sensor nodes increase their transmission power to send data packets directly to base station (single step), while multi-step communication uses normal data packets. Sensors with normal data packets cannot send query-based or critical data packets until the well has not received them. In multi-step communication, a path with a smaller number of steps is selected when there are two or more paths. If two or more stepping nodes have the same number, then the neighbor node is selected with the lowest energy consumption. It defines a threshold to control the rise in temperature, and if the temperature of the node exceeds the desired threshold, it eliminates all paths along with the neighboring node. If the temperature of the node reaches the threshold after receiving the data packet, it sends the packet back to the previous node and the previous node would be assigned as a hotspot. This algorithm has a good performance in detection rato, but they did not pay attention to the reliability and energy consumpation.

Chze and Leong [27] provided a Secure Multi-Hop Routing Protocol (SMRP) for communications over WSNs. The applied technique in this solution is to authenticate users based on a multi-layered security architecture. In this system, nodes must be authenticated before connecting to the network. A multilayer parameter is used in the routing algorithm, which means that users must be authenticated to use the route. This algorithm is capable of resisting attacks such as Greyhole, Black hole, Sink hole, and spoofing. However, one of the problems is the inefficiency of the routing algorithm in controlling large networks. Therefore, the security architecture of this system has a good efficiency while the provided routing algorithm is not suitable to be used in the Internet of Things systems. In this paper, the authors found a high security architecture low delay and high bandwidth. However, this algorithm does not pay attention to the energy consumption and large networks.

Guleria and Verma [28] proposed protocol achieves optimal clustering using a weighing function for $\mathrm{CH}$ selection. Cluster formation is based on a number of factors such, including node remaining energy, Received Signal Strength Indicator (RSSI) and node density. Priority weights are also allocated to these metrics. For each round, the $\mathrm{CH}$ with the highest likelihood will be chosen as the best $\mathrm{CH}$. The aim of this article is to propose an energy-efficient Load Balanced Cluster-Based Routing Protocol based on Ant Colony Optimization (LB-CR-ACO), which will increase the lifespan of WSN networks. Simulation results shows that the method decrease energy consumption and increase the network lifetime. Nevertheless, the proposed method does not have high throughput.

Altakhayneh, et al. [29] proposed Genetic Low Energy Adaptive Clustering (G-LEACH) for solving the $\mathrm{CH}$ selection and routing in WSN. The reason of this paper is to simulate the utilize of different routing methods in modern wireless communication, especially in WSN communication. The second is G-LEACH, which is a more advanced version 
of LEACH techniques that makes use of genetic algorithm. In this comparative simulation, both the LEACH and GLEACH algorithms try to find the best $\mathrm{CH}$ in order to increase packet delivery to the $\mathrm{CH}$, cluster life time, and network life time. However, this algorithm suffers from throughput and low load balancing.

PSO-ECHSrk is an energy-efficient cluster head selection algorithm proposed by Rao, et al. [30]. It is based on particle swarm optimization (PSO). The algorithm is built around an effective particle encoding and fitness function scheme. $\mathrm{CH}$ selection and cluster formation are the two steps of the algorithm. PSO is used to choose the $\mathrm{CH}$, also the residual energy, distance parameters are used in the $\mathrm{CH}$ selection algorithm. All sensor nodes send their location and residual energy to the base station during the $\mathrm{CH}$ selection phase to see if they satisfy the threshold energy to be considered for a $\mathrm{CH}$. In this paper, the authors found a good efficiency in energy consumption. However, this method has limited functionality in low reliability and low scalability.

Baskaran and Sadagopan [31] proposed hybrid firefly algorithm. Using crossover and mutation in the suggested algorithm, the best fireflies chosen through tournament selection are allowed to replicate among themselves. The proposed method allows for faster convergence while also avoiding multiple local optima. The suggested algorithm has a high efficiency in terms of packet loss rate and network lifetime, according to simulation results. However, author does not pay attention the delay and rate of monitors.

Karaboga, et al. [32] using an Artificial Bee Colony (ABC) algorithm, and present a new energy-efficient clustering mechanism. The cluster heads are determined using ABC algorithm, where each solution represents an array of objects, which contains a sensor node. The ABC uses a population of bees to locate cluster-heads in the search space. $\mathrm{CH}$ selection fitness is expressed as a fitness value that is inversely proportional to the amount of energy consumed during a tour. The simulation results show that the $\mathrm{ABC}$ routing protocol can successfully optimize network lifetime and reduce transfer delays. However, this method suffer from low packet delivery rate and the node makes independence decisions without any centralized control. In table, 1 shows the advantage and disadvantage of the algorithms.

Table 1. A side-by-side comparison among the investigated methods.

\begin{tabular}{|c|c|c|c|}
\hline Author & Method & Advantage & Disadvantage \\
\hline \multirow{2}{*}{ Deepa and Latha [23] } & Hybrid Hierarchical Secure Routing & \multirow{3}{*}{$\begin{array}{l}\text { Increase reliability } \\
\text { Increase number of packets received } \\
\text { High throughput -Low energy } \\
\text { consumption }\end{array}$} & Low load balancing \\
\hline & Protocol (HHSRP). & & Low mobility \\
\hline Sharma, et al. [24] & Efficient Routing Algorithm (ERA) & & Low reliability Service monitoring \\
\hline B. D and Al-Turjman [25] & $\begin{array}{l}\text { Authentication and Encryption } \\
\text { Model (ATE). }\end{array}$ & Detection rato & Low reliability -High energy consumpation. \\
\hline Chze and Leong [26] & $\begin{array}{l}\text { Secure Multi-Hop Routing Protocol } \\
\text { (SMRP) }\end{array}$ & $\begin{array}{l}\text { High security architecture } \\
\text { Low delay } \\
\text { High bandwidth }\end{array}$ & $\begin{array}{l}\text { High energy consumption - Not support large } \\
\text { networks }\end{array}$ \\
\hline Guleria and Verma [27] & $\begin{array}{l}\text { Received Signal Strength Indicator } \\
\text { (RSSI) }\end{array}$ & $\begin{array}{l}\text { Decrease energy consumption } \\
\text { Increase the network lifetime }\end{array}$ & Low throughput \\
\hline Altakhayneh, et al. [28] & $\begin{array}{l}\text { Genetic Low Energy Adaptive } \\
\text { Clustering (G-LEACH) }\end{array}$ & $\begin{array}{l}\text { High packet delivery rate } \\
\text { High energy of } \mathrm{CH}\end{array}$ & Low throughput-Low load mobility \\
\hline Rao, et al. [29] & PSO-ECHSrk & Low energy consumption & Low scalability \\
\hline Baskaran and Sadagopan [30] & firefly algorithm & Network lifetime & High delay \\
\hline
\end{tabular}

\section{Statement of the Problem}

The challenge of energy constraints in WSNs can be significantly overcome using clustering methods. Scalability is one of the most crucial aspects of WSNs. Scalability and extending the life of WSNs can be achieved by clustering. In this method, the $\mathrm{CH}$ can be replaced or removed, the controller is responsible for network management. The controller is the connection between the $\mathrm{CH}$ and the base station. The following are the advantages of clustering in WSNs, and Figure 3 shows the clustering:

Reduction of the routing table size: Routing in each cluster is done locally by clustering. This reduces the size of the routing table.

Savings in the consumed bandwidth: Extracommunication is reduced by clustering and ultimately saves communication bandwidth.

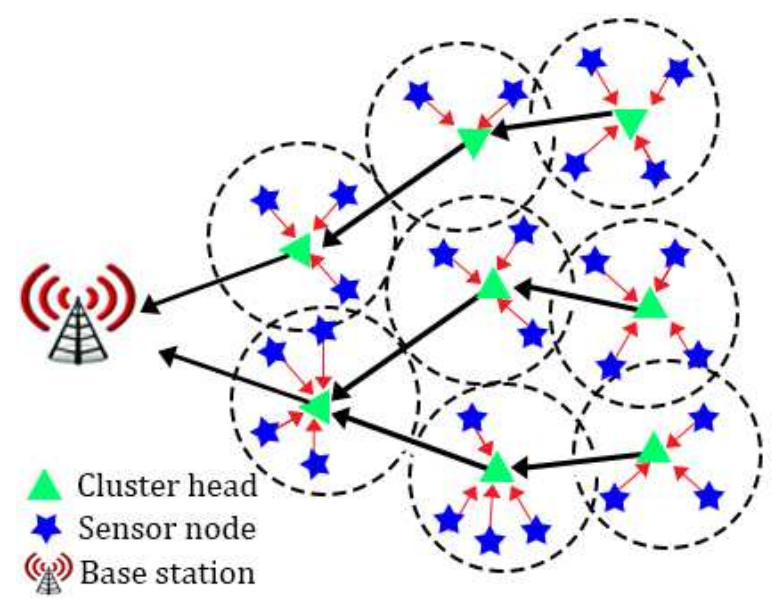

Figure 3. Clustering method and sending data to the sink [15]. 
The method that transfer the data between the sensor and where the base station is observed called routing. On the one hand, routing can be considered as a method of data transfer between sensor nodes and on the other hand, information transfer between sensor nodes within the network and the final base station can be defined as routing [33]. A very simple method to do this task is that each sensor send data directly to the base station. Nevertheless, direct-sending to base station is very costly because the nodes are may far from the base station and it cause evacuated early, which this method has limits the life of the network [34]. This issue is important especially when sensors are arranged to cover a large geographic area, or where the sensors are moving and may move away from the base station:

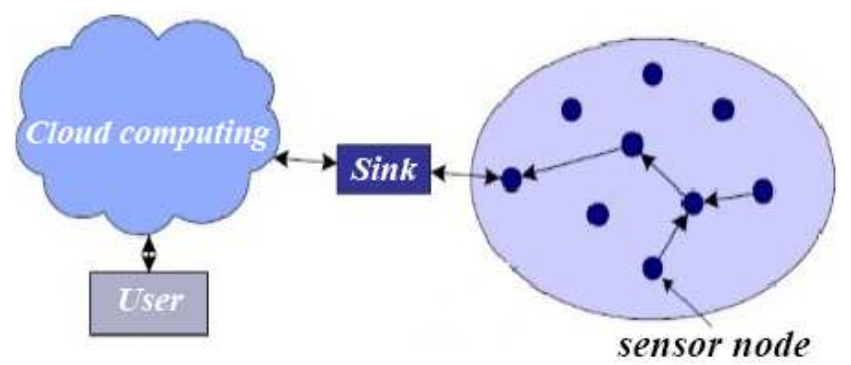

Figure 4. Architecture of a wireless sensor network [35].

Wireless sensor network in fixed SDN-WSN

SDN controller is as a gateway in the centralized SDNWSN network that manages all sensors, including sensor behaviors, sleep nodes, routing path, and data transmission [36]. The topology of the SDN-WSN network can be both fixed and mobile. The controller uses a middleware to control information, tables, thresholds, mapping performance, and information. The SDN controller, which manages all nodes and the network management unit is located in the base station. A controller that monitors the whole network consists of the control layer. The application layer of SDN-WSN includes various applications such as routing. Figure 5 indicates the performance of a SDN-WSN as well as the advantages of centralized mode:

The SDN controller is suitable for small networks, which have a duty to manage all of the sensors.

The load balancing mechanism is used to store the energy of the nodes.

The network is more balanced, and the area's nodes are managed by the regional controller.

The $\mathrm{CH}$ is responsible for data transfer in the network, and it sends data to the sink via the main controller.

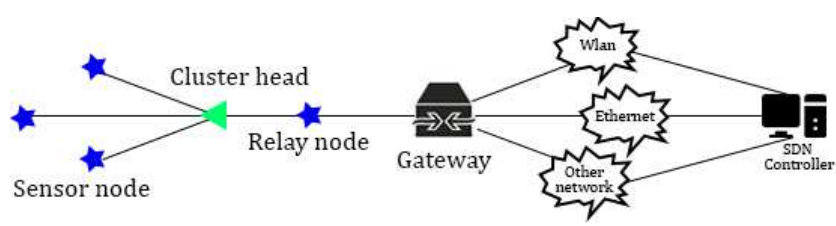

Figure 5. Architecture of wire sensor networks in fixed software-defined wireless sensor networks (SDN-WSN) [37].
The whole network nodes are divided into areas according to the distributed SDWSN model. There is a $\mathrm{CH}$ in each area that is the main controller located at the gateway. The controllers manage and synchronize the sensors and $\mathrm{CH}$. Figure 6 shows the performance.
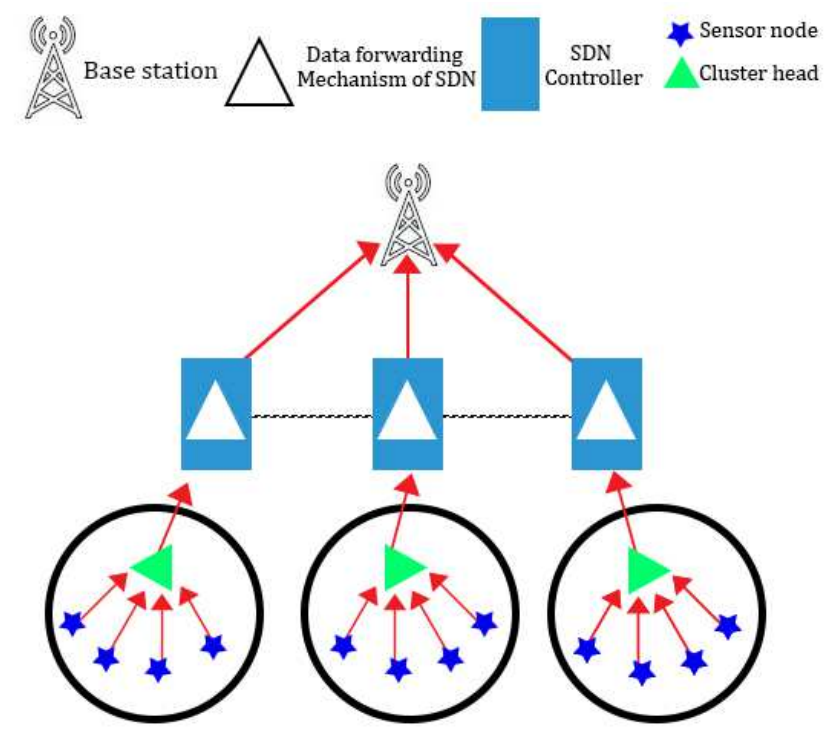

Figure 6. Architecture of Wireless sensor network in distributed SDN-WSN [38].

There are 2 types of topologies in this type of network, $\mathrm{CH}$ node and normal nodes under SDN controller. Central nodes act as transmitters for data transmission, while normal nodes are used only to receive data flow. Finally, a Network Operating System (NOS), which is responsible for the entire network such as topology management. Distributed SDNWSN is suitable due to the high data transfer to the network and high load balanced for large-scale WSN. The advantages of SDN-WSN distribution include the following:

Easy expansion of network topology. Nodes can be added or removed easily in the area.

Management of the controller easier than other conventional nodes.

The transmitted messages in non-SDs based on WSN are trusted, because they are sent by each node to identify neighbor nodes. This method adds significant overhead to the network and also consumes a lot of energy. Each node must store routing tables in limited memory and calculate the path for the other nodes to execute these decisions. Many of the tasks of the resource-hungry are transmitted to the controllers in a SDN-WSN because it has a power supply and examines the entire network. The nodes should not constantly send the message to discover the topology in SDN-WSN [39]. The controllers in the SDN-WSN make the routing decision. Therefore, nodes do not need to store routing information within their routing diagrams. In addition, controllers can adjust the range of each node to reduce the reference information between the nodes. Implementation of these tasks by the controller in SDN-WSN can store the remaining energy of the nodes [40]. 
Service quality parameters

Packet Delivery Ratio (PDR): This ratio is based on the sum of the total received packets in to the sink to the total sum of generated packets at all sensor nodes in a network, which shows the percentage of total packages delivered in the sink [41].

$$
\text { Packet delivery rate }=\frac{\sum \text { No.of packets recieved }}{\sum \text { No.of packets sent }} * 100 \% \text { (1) }
$$

Throughput: it is defined as the number of packets delivered to the sink per unit time. The measurement power is significant towards the computational efficiency.

$$
=\frac{\sum \text { No.of packets sent } * \text { packet size }}{\text { Time taken }} \text { Throughput }
$$

Live nodes: Nodes in the network have enough energy to process their tasks and can send their data to the $\mathrm{CH}$.

$$
N_{A}^{i}=E_{\text {Res }}^{i}(n)>0
$$

In which, $E_{\text {Res }}(n)$ is the residual energy of the node in each round.

Network lifetime: it describes the long life of a network for the number of rounds at a given time. In other words, it shows how long or how many rounds he network can operate. Finally, efforts should be made to extend the life of the network, so that data packets in the BS can be transmitted for longer.

$$
\text { Network life time }=\min _{\mathrm{j}}=\left[\frac{\sum i=1 C M_{i j} * L_{i}}{N_{j}}\right]
$$

In this equation, $C M_{i j}$ is the coverage matrix and $\mathrm{L}_{\mathrm{i}}$ is the energy of sensor nodes, and $\mathrm{N}_{\mathrm{j}}$ is the sensors in the network.

Consumed Energy: An open space model (fs) is used for energy consumed by sensor nodes in data transmission, when the distance is less than the threshold value $\mathrm{d}_{0}$, otherwise, the multi-routing (mp) model is used. The energy consumed by the sensor is calculated to transmit L-bit information at distance $d$ so that $E_{\text {elec }}, E_{\mathrm{fs}}$, and $E_{\mathrm{mp}}$ are the consumed energy by the electronic device and by the amplifier in open space and multi-way space, respectively.

$$
\mathrm{F}(\mathrm{x})=\left\{\begin{array}{l}
L E_{\text {elec }}+L E_{f s} d^{2} \text { if } d<d_{0} \\
L E_{\text {elec }}+L E_{m p} d^{4} \text { if } d>d_{0}
\end{array}\right.
$$

Moreover, the consumed energy in generating bit control messages is calculated according to Equation $6 . \mathrm{E}_{\text {crtl }}$ depends on various factors such as digital encryption, modulation, filtering and signal propagation and so on.

$$
\mathrm{E}_{\mathrm{CTRL}}(\mathrm{L}, \mathrm{d})=\mathrm{LE}_{\mathrm{ctrl}}
$$

Selecting a CH by Game of Theory:

A. Identification of nodes by the controller:

At first, the controller knows nothing about the network. Therefore, nodes must send their information such as address, location, residual energy, and their types to the controller.
B. The controller starts the strategy of choosing the game theory algorithm:

When the goal is to select a number of nodes as $\mathrm{CH}$, the clustering game is played by network nodes, which corresponds to selecting at least one $\mathrm{CH}$ from the node population. The $\mathrm{CH}$ is responsible for data collection from other nodes and sending after compressing proportional to another node. In this method, the proposed method is sent to the controller. The game is defined as $\mathrm{FB}=\langle\mathrm{N}, \mathrm{T}, \mathrm{E}>$, where $\mathrm{N}$ is the set of nodes $\mathrm{T}$, the set of available strategies, and $\mathrm{E}$ is the set of utility functions of the nodes.

$$
\begin{gathered}
\mathrm{FB}=<\mathrm{N}, \mathrm{T}, \mathrm{E}> \\
\mathrm{N}=\text { nodes, } \mathrm{T}=(\mathrm{Ti}), \mathrm{E}=(\mathrm{Ei})
\end{gathered}
$$

Players are sensors, there are $\mathrm{N}$ nodes in the network. The node selection strategy can have two functions.

C. Selection or not selection of a node as a $\mathrm{CH}$

A sensor decides to announce itself as a $\mathrm{CH}$, or other nodes can be introduced as a $\mathrm{CH}$. The symbol $\mathrm{D}$ is considered as the Declare strategy as the caption and the ND is considered as the symbol of Not Declaring itself as the CH. Strategy space is defined as follows:

$$
\mathrm{S}=(\text { Declare}, \text { Not Declare })=(\mathrm{D}, \mathrm{ND})
$$

D. The reward of selecting or not selecting cluster head

According to rewards, if a node does not send own information, to the $\mathrm{CH}$, the $\mathrm{CH}$ reward will be zero. In other words, if the node has to send own data directly to the controller, which will consume a lot of energy. If at least one node identifies node in the area as $\mathrm{CH}$, its score will be $\mathrm{W}$. This means that delivery of data to the controller is sent by $\mathrm{CH}$. If the one node introduces itself as a $\mathrm{CH}$, its reward to deliver data through clustering is reduced by an equal value at the cost of being a $\mathrm{CH}$. In this case, the final reward will be W-D. It is clear that the game is symmetrical. Since the reward depends only on the players' strategies, because of that no specific node is considered. This means that each node can select itself as a CH with reward. Strategy $(\mathrm{D}, \mathrm{D})$ is not a Nash equilibrium. For this reason, it is best for each player to change the strategy to ND, in which case the reward will be $\mathrm{W}(\mathrm{W}>\mathrm{W}-\mathrm{D})$.

Similarly, strategy (ND, ND) it is not a Nash equilibrium so that each node prefers to change direction and introduces itself as a $\mathrm{CH}$ because this will lead to a positive reward. If the first player selects to be a $\mathrm{CH}$ and the second player selects to be a normal node, then neither of them has an incentive to change their choice. Hence, the strategy set (ND, D) is an equilibrium Nash. Similarly, (D, ND) is also an equilibrium Nash. Although these two strategies (D, ND) and (ND, D) are Nash equilibrium, there is no symmetrical Nash equilibrium in this game because there is no normal strategy for all players with an equilibrium result. In the following, useful expressions and theories that correspond to the clustering game with $\mathrm{N}$ players will be mentioned. 
Table 2. Rewards of two nodes for clustering game.

\begin{tabular}{|c|c|c|c|}
\hline Second player strategy & First player strategy & Declare the node as cluster head & Not declare the cluster head \\
\hline Declare the node as cluster head & & W-D, W-D & W, W-D \\
\hline Not declare the cluster head & & W, W-D & $(0,0)$ \\
\hline
\end{tabular}

$\mathrm{T}$ vector is considered to represent the strategies that players follow to extend the game from two nodes to $\mathrm{N}$ players. If no player considers itself as a $\mathrm{CH}$, then the reward for all players will be zero. If at least one player, called k, plays $\mathrm{D}$, then the score of all players except $\mathrm{k}$ will be equal to $\mathrm{w}$, while the cost of introducing itself as a $\mathrm{CH}$ will be deducted from the initial reward $(\mathrm{w})$ of player $\mathrm{k}$. Therefore, the utility function $U_{i}(S)$ for the optional player $i$ is as follows:

$$
\mathrm{U}_{\mathrm{i}}(\mathrm{s})=\left\{\begin{array}{c}
0, \text { if } T_{j}=N D, \forall j \in N \\
W-D, \text { if } S_{i}=D \\
\text { v, if } S_{i}=N D \text { and } \exists j \in \mathrm{N} S_{j}=D
\end{array}\right.
$$

If a node is not selected as a $\mathrm{CH}$, the game continues and another node is declared as a $\mathrm{CH}$.

E. Another round of the game and introduction a cluster head

If the reward of the nodes to the controller is zero, the game will be played again in another round

F. Do the other nodes send their own information to the $\mathrm{CH}$ or not?

$\mathrm{G}$. If the $\mathrm{CH}$ gets all information from all nodes of the area, the $\mathrm{CH}$ throughput and consumed energy will be calculated.

Declaring or non-declaring of a node as a $\mathrm{CH}$, the two numbers $\mathrm{w}$ and $\mathrm{D}$ cannot be the only $\mathrm{CH}$ criterion. As mentioned, a very important issue in WSN is the limited resources, especially the energy source of the nodes. A node that is introduced as a $\mathrm{CH}$ in the clustering structure will lose a lot of energy. Therefore, it is better to have $\mathrm{CH}$ candidate that have more energy than other nodes. They should have more throughput to be able to send information to other $\mathrm{CH}$ or controller with a high percentage. The desired changes are applied to some extent by multiplying the remaining energy of the node by the output of the utility function. The node that has more residual energy, as well as better throughput, will have more points to enjoy by implementing this change. The connection is obtained from the following relation and is placed in the neighborhood table to gain operational capability.

$$
\operatorname{Link}_{\mathrm{R}}=\mu \mathrm{N}_{\text {succ }} / \mathrm{N}_{\text {total }}+(6-\mu) \operatorname{Link}_{\mathrm{R}}
$$

The weighted mean factor coefficient is used in $8 \mu$ in the proposed algorithm is the number of packets that succeed node $\mathrm{N}_{\text {succ }}$ neighbors is considered as much as 0.4 . The $\mathrm{N}_{\text {total }}$ parameter is the total number of packets sent to neighbor node. This equation consists of two parts, the first part whose coefficient $(\mu)$ is related to the last time period, and the second part, whose coefficient $(\mu-1)$ is related to the time periods before the last time period. According to the equation, it is obvious that the more time intervals, the greater the effect of the last time period in calculating the throughput of the link. The desired changes are applied to some extent by multiplying the remaining energy of the node and the operating power by the output of the utility function. The node that has the remaining energy and throughput will also have more points to enjoy by implementing this change.

$$
\mathrm{E}_{\mathrm{i}}(\mathrm{T})\left\{\begin{array}{c}
0, \text { if } T_{j}=N D, \forall j \in N \\
\text { (Remining energy of } \left.T_{j}\right) *(W-D), \text { if } T_{j}=D \\
\left(\text { Throughput of } T_{j}\right) *(W-D) \text { if } N=D \\
\text { Remining energy of } T_{j} * \text { Throughput of } T_{j} * E \text {, if } T_{j}=N D \text { and } \exists j \in N T_{j}=D
\end{array}\right.
$$

H. Calculation of the scores of all nodes

In the proposed scheme, this choice is implemented completely randomly and with a random function. Using probability may create problems for the proposed scheme. The node scores are calculated before the clustering operation to reduce the probability effects in the proposed method. The total score of all nodes is calculated each time the game. Obviously, the best network mode is when the score is higher. In each round of the game $(\mathrm{P})$, the selected node declares itself as a $\mathrm{CH}$. The points are obtained for each node based on the modified utility function. These points must be added together. If the score is better and higher, the $\mathrm{CH}$ has selected otherwise another round of game will be started to find the best $\mathrm{CH}$. The following equation shows this.

$$
\operatorname{Sum}_{\mathrm{p}}=\sum_{i=1}^{N} U_{i}(s)
$$

If the $\mathrm{CH}$ node cannot receive information from the node, it would be identified.

Zero reward is given to $\mathrm{CH}$.

Is the node not sending the information to the $\mathrm{CH}$ farther away from the $\mathrm{CH}$ ?

First, each controller called $\mathrm{Vi}$ sends a radio message to $\mathrm{u}$ unknown node and records the time ti to receive the answer.

Each Vi sends a radio message with ti to an unknown node.

The controller calculates the distance of each reference to $\mathrm{u}$ from ti and sums the squares by subtracting the $\mathrm{u}$ coordinates. 


$$
\left.\left.\epsilon=\sum\left(d b_{i}-\sqrt{\left(x-x_{i}\right.}\right)^{2}+\sqrt{\left(y-y_{i}\right.}\right)^{2}\right)^{2}
$$

The controller tests whether the coordinates are acceptable or not.

The controller can now perform two tests to see if the $U$ coordinates are acceptable. Test $\mathrm{a}$ is that the controller obtains the distance between each estimated Vi and $\mathrm{U}$ and compares it with the distance obtained from the protocol. If they differ greatly, the estimate is unacceptable and the node must be far from the geographical environment, but the test $b$ is that $\mathrm{u}$ must be at least among the three sent nodes, otherwise it is not acceptable. If both tests $a$ and $b$ are positive, then the distances are constant with the estimated position and the node falls into a triangle of references. Therefore, the controller can assume that the estimated position of node is in the correct geographical position. However, if one of the tests fails instead, then the available information is not enough to examine the data environment. The estimate does not pass test a and is fixed far away from the environment. In fact, a delayed response node can pretend that the node is far from the environment.

$$
\mathrm{U}=\left\{\begin{array}{c}
\left(y-y_{i}\right)^{2}+\left(x-x_{i}\right)^{2}<R^{2} \\
\left(y-y_{i}\right)^{2}+\left(x-x_{i}\right)^{2}>\left(y_{0}-y_{i}\right)^{2}+\left(x_{0}-x_{i}\right)^{2}
\end{array}\right.
$$

$r$ meter of the signal that is sent

$\mathrm{X}_{0}$ and $\mathrm{Y}_{0}$ are the actual coordinates of $\mathrm{u}$, which the controller checks again.

If the node is farther from the Euclidean distance, it is turned back and the other round of the game is played to select the spark.

If the selected node is close, the command to send data to the $\mathrm{CH}$.

After all the nodes have recognized the $\mathrm{CH}$ and the $\mathrm{CH}$ score is higher, the $\mathrm{CH}$ is definitely selected and they will send the data to the controller.

The controller takes the information from $\mathrm{CH}$ 'S and sends to the sink.

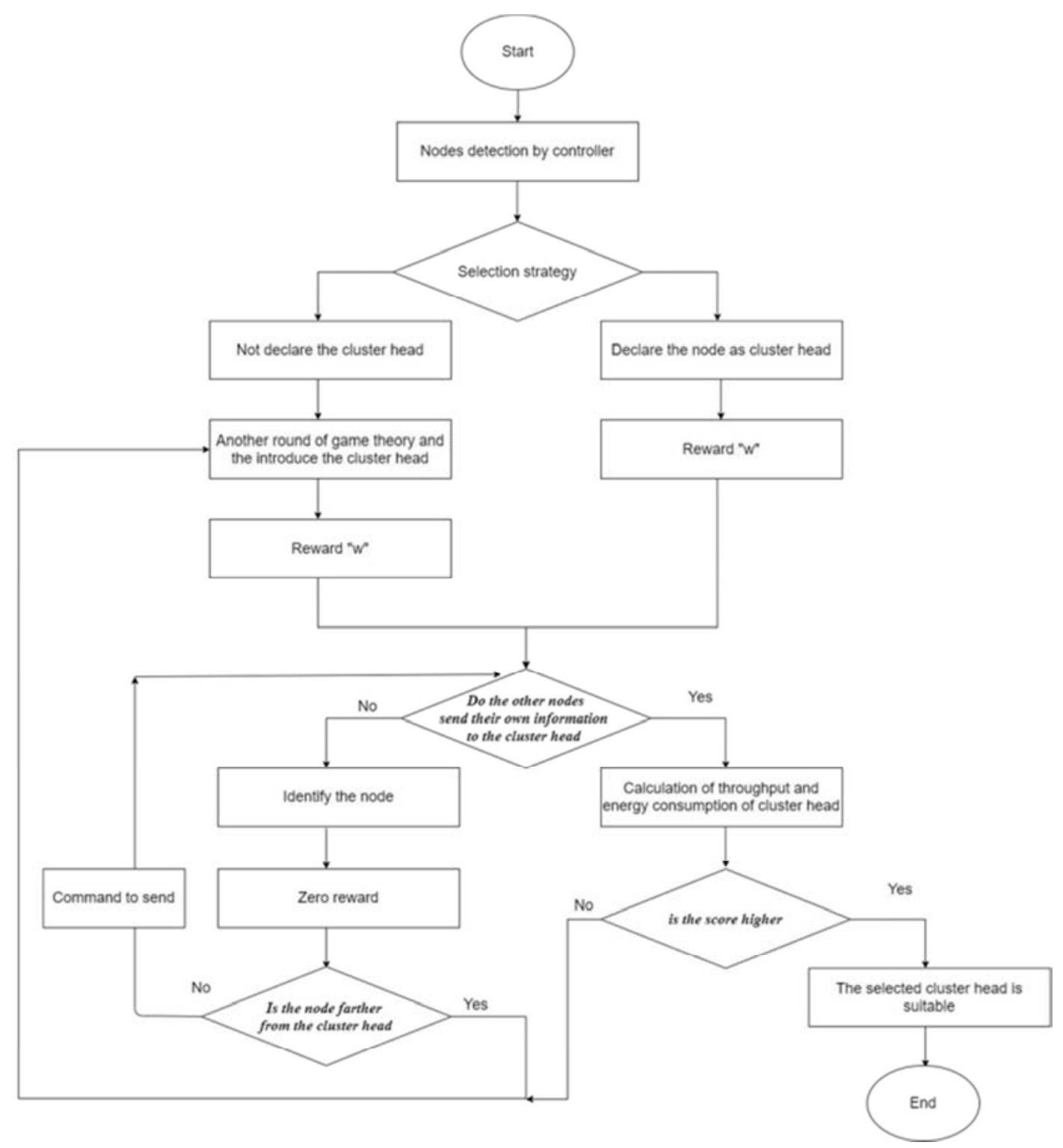

Figure 7. Flowchart of $\mathrm{CH}$ selection via Game theory. 
Routing with Game Theory:

Identification of nodes by the controller

At first, the controller knows nothing about the network. Therefore, nodes must send their state information such as location, residual energy, ID and their types to the controller.

Selection of the $\mathrm{CH}$ using the game theory algorithm

The $\mathrm{CH}$ is selected using the above proposed algorithm.

Preparing the package of each node to send

After identify, the nodes prepare the packets and become ready to be sent.

High and low prioritization is considered in data transmission.

Sending data using FIFO priority algorithm

First, high-priority queues are served. Thus, highpriority traffic classes will have little delay. A new scheduling plan is announced for high-priority incoming traffic, which uses a transit queue buffer and a sending queue buffer to alternately select packets. When the prioritized package is served over a period of time, the counter is reduced by the size of the package. If a queue fails to send a packet in the previous period, the counter value is retained and used in the next time. Therefore, if a service queue receives less than the amount sent in one period, it will have the opportunity to receive more service in the next period.

Discovering neighboring nodes

If two nodes are neighbors in a radio range and can interact directly with each other, an edge is considered between them. $\mathrm{N}_{\mathrm{i}}$ is a set containing all the neighbors of node i representing the number of nodes in the network.

The network player selects the node according to the next node conditions

In the routing problem, the source node can be considered as a player. The set of operations is all the states of the path that exist from origin to destination. In WSN, intermediate nodes play an important role in routing. Since WSN have limited power consumption, they may be reluctant to participate in the middle role. In other words, if all nodes do not want to use energy as an intermediate node, the efficiency of the network will decrease. For this reason, WSNs sometimes operate in the form of optional resource sharing networks.

$$
\begin{gathered}
\alpha j(s)=\alpha j\left(\sum i € N \text { i } \neq j \text { Si }\right) \\
U j(s)=\alpha j(s)+\beta j(s)
\end{gathered}
$$

Benefits come from sharing the resources of a user with others $\beta \mathrm{j}(\mathrm{sj})=\beta \mathrm{j}(\mathrm{s})$. Benefit (cost) is the result of sharing one's interests with others. $\mathrm{S}=0$ means subscription and $\mathrm{S}=1$ means non-subscription.

The player is the network that decides which neighbor to receive the package. In this proposed method, a node is a player that defines its maximum profit with the shortest path and the lowest energy consumption according to the equation, creates a table like the following table based on the number of neighboring nodes, and completes it from the characteristics of neighbors. Each node has neighbor characteristics, i.e. geographical distance to the earthenware and its amount of energy. An example of a created table:

\begin{tabular}{|c|c|c|c|}
\hline $\begin{array}{ll}\text { Next node } & \text { Node } 1 \\
\end{array}$ & $B_{1}$ & $B_{2}$ & $B_{3}$ \\
\hline$A_{1}$ & $(2,40)$ & $(2,46)$ & $(46,4)$ \\
\hline$A_{2}$ & $(36,6)$ & $(42,5)$ & $(46,6)$ \\
\hline$A_{3}$ & $(48,8)$ & $(56,10)$ & $(56,4)$ \\
\hline
\end{tabular}

Table 3. Sample of routing matrix in terms of distance energy.

Therefore, the next node A3 is selected because it is the shortest distance with an approximate distance of $10,8,6,5$, 4, 2 from the well and different energies among the maximum available energies.

Referring to node interaction

$H_{i}(t)=\left(h^{j} I(t)|j=1, \ldots,| N_{i} \mid\right)$ is the history of interaction (transaction) of node I with neighbors. For example, the behavior of nodes belonging to $\mathrm{Ni}$ at time $\mathrm{t}$ occurs in packets. $\mathrm{R}$ ji $>\mathrm{Hji}(\mathrm{t})=\angle \mathrm{Fji}$ is the history of the interaction of node $\mathrm{i}$ on node $\mathrm{j}$. Fji is the number of packets that node $\mathrm{j}$ precedes for node $\mathrm{i}$ and $\mathrm{R}_{\mathrm{ji}}$ is the number of packets that node $i$ requested from node $j$ to send to at time $t$. $\Theta i$ is the private information of node $i$ about other nodes without those nodes knowing the details. This information plays an essential role in assessing the correct amount of nodes. Therefore, a database is considered for each node, and each input of this database retains the properties of one of the neighboring nodes; for example, node properties, the correct value estimated for the node, and its history of interactions. The correct value that node $\mathrm{i}$ estimates for node $\mathrm{j}$ is denoted by $\mathrm{T}_{\mathrm{ij}}$, which is equal to the following equation.

$$
\mathrm{T}_{\mathrm{j}}^{\mathrm{i}}=(1-\alpha) T \mathrm{Ti}^{\mathrm{j}, \mathrm{g}}+\alpha \mathrm{T}_{\mathrm{i}}^{\mathrm{j}, \mathrm{o}}
$$

In this $\mathrm{Ti}^{\mathrm{j}, \mathrm{g}}$ is the value of the predicted value of node $\mathrm{j}$, and node $i$ is obtained based on game analysis on the properties of node $j$. To calculate this value, node $i$ must calculate the expected profit from the game of node $j$ during the game with its neighbor node (node $j$ ) as follows.

$$
\mathrm{T}^{\mathrm{i}, \mathrm{g}} \mathrm{j}_{\mathrm{g}} \mathrm{U}_{\mathrm{j}}^{\mathrm{i}} / \sum\left(\mathrm{U}_{\mathrm{j})}^{\mathrm{i}} \mathrm{j} \in \mathrm{N}_{\mathrm{i}}\right.
$$

$\mathrm{T}^{\mathrm{i}, \mathrm{g}}$ is the value that calculates node $\mathrm{i}$ based on direct observations of the transaction with node $\mathrm{j}$ according to the following equation.

$$
\mathrm{T}^{\mathrm{i}, \mathrm{g}}{ }_{\mathrm{j}, \mathrm{O}}=\mathrm{F}_{\mathrm{j}}^{\mathrm{i}} / \mathrm{R}_{\mathrm{j}}^{\mathrm{i}}
$$

$\alpha$ is a factor that indicates the amount of priorities. If $\alpha=0$, the transaction history of the nodes is not considered and it is assumed that the nodes have not had any previous transactions with each other. As the set up time of the network increases, the value of $\alpha$ also increases too. 


\section{Calculation of weight factor $\alpha$}

The weight factor plays an important role in calculating the mean $\mathrm{T}_{\mathrm{j}}^{\mathrm{i}}$. Suppose the number of transactions between nodes $i$ and $\mathrm{j}$ is equal to $\delta(\mathrm{j}, \mathrm{i})$. As a result, the weight factor is calculated as follows:

$$
\mathrm{A}=\frac{\delta(\mathrm{i}, \mathrm{j})}{\sum j \epsilon N i \delta(\mathrm{i}, \mathrm{j})}
$$

Nodes can estimate the correct value for them during their transactions with neighboring nodes when the expressions in the above equation are known.

$\mathrm{T}_{\mathrm{j}}^{\mathrm{i}}=$ According to the definition this function is calculated based on observations of node interaction history. Suppose node $\mathrm{i}$ calculates the correct value for node $\mathrm{j}$ for the kth time after the node is set up, so it must use the k-1th observations and previous interaction history.

$$
\mathrm{T}_{\mathrm{j} o}^{\mathrm{i}}(\mathrm{k})=\mathrm{F}_{\mathrm{j}}^{\mathrm{i}}(\mathrm{k}-1) / \mathrm{R}_{\mathrm{j}}^{\mathrm{i}}(\mathrm{k}-1)
$$

In which, $\mathrm{F}_{\mathrm{j}}^{\mathrm{i}}(\mathrm{k}-1)$ is the interaction history after the evaluation of $k-1$ th correct value.

\section{Estimation of profit from routing}

In this game, the energy distribution of player $i$ has a functional space according to $<\mathrm{a}_{11}, \mathrm{a}_{\mathrm{i} 2}>$, where $\mathrm{a}_{\mathrm{i} 1}$ is the amount of personal energy and $\mathrm{a}_{\mathrm{i} 2}$ is the amount of energy in front of the packages. It is clear that $\mathrm{a}_{\mathrm{i} 1}, \mathrm{a}_{\mathrm{i} 2}$ must be true in the condition $\mathrm{Ei}>\mathrm{a}_{\mathrm{i} 2}+\mathrm{a}_{\mathrm{i} 1}$. The profit function of Node $\mathrm{i}, \mathrm{u}_{\mathrm{i}}=$ $\left(\mathrm{a}_{01}, \mathrm{a}_{02}\right),\left(\mathrm{a}_{11}, \mathrm{a}_{12}\right) ; \theta$ is defined as follows:

In this equation, $\mathrm{j}=1-\mathrm{i}, \gamma+\beta=1$, and $\gamma \mathrm{j}$ and $\mathrm{x}_{\mathrm{i}}$ are constant, which means that the previous profits in personal energy and frontal energy are also present and effective. The condition $\mathrm{E}_{\mathrm{i}}>\mathrm{a}_{\mathrm{i} 2}+\mathrm{a}_{\mathrm{i} 1}$ must be true after simplifying the profit function.

$$
\mathrm{L}_{\mathrm{n}} \mathrm{u}_{\mathrm{i}}=\beta \ln \left(\mathrm{x}_{\mathrm{i}}+\frac{a i 1}{1+a j 1}\right)+\gamma \mathrm{L}_{\mathrm{n}}\left(\gamma_{\mathrm{i}}+\frac{\Theta \text { iai2 }}{1+\Theta \text { jaj } 2}\right)
$$

For this reason, the logarithm function is used. This function has a small growth and invertible. Then, $\mathrm{U}_{\mathrm{i}}=\mathrm{L}_{\mathrm{n}} \mathrm{u}_{\mathrm{i}}$ in node $\mathrm{i}$ does not know the type of node $\mathrm{j}$, but the probability density function of the type of node $j$ is $\Theta$ (f). As a result, the expected gain of node $\mathrm{i}$ is equal to the mathematical expectation of $U_{i}$.

$$
\mathrm{EU}_{\mathrm{i}}=\mathrm{f}(\Theta j) \mathrm{Ui} \mathrm{d} \Theta \mathrm{j}
$$

Considering the Lagrange function, the initial condition is:

$$
\mathrm{L}_{\mathrm{i}}=\mathrm{EU}_{\mathrm{i}+} \Lambda\left(\mathrm{E}_{\mathrm{i}}-\mathrm{a}_{\mathrm{i} 2}-\mathrm{a}_{\mathrm{i} 1}\right)
$$

As a result, the expected profit of node $i$ is represented by $\mathrm{U}$, which is obtained by simplifying the Lagrangian equations that can be used to calculate the value of $T_{i}$. If the desired profit is obtained, the algorithm will continue to work. Otherwise, the network player considers another node.

Data storage of nodes

$\Theta_{i}$ is the private information of node $i$ about other nodes without those nodes knowing the details. This information plays an essential role in assessing the correct amount of nodes. Therefore, a database is considered for each node. Each input of this database holds the properties of one of the neighboring nodes. For example, the properties of the node, the correct value for that node, its history of interactions, and the benefit gained from using the information in future routing.

When the node with a lower priority wants to send the data to the header.

Identify adjacent nodes

Receiving the list of neighbors in the proposed method is done through all the broadcasts of Hello packages. This package contains node information (including ID, residual energy, and node location).

Estimating node distances with received signal strength

The distances between nodes can be estimated by the strength of the received signal. Therefore, it is assumed that network nodes are aware of their position relative to their neighbors based on the estimated signal strength. The shortest and closest node is selected.

Calculation of the energy consumption of the next node

After selecting the node, the energy is calculated to see if the next node has good energy consumption or not. If the node has the desired energy, data is sent, otherwise another node is identified. The calculation of consumed energy of the proposed model is based on the LEACH algorithm. The amount of energy required for this transfer operation to occur in the sending node is calculated according to the following equation according to the algorithm for transferring a data packet of $\mathrm{k}$ bits to the distance $\mathrm{i} d$ (the distance between the data sending node and the data receiving node):

$$
E_{t x}(i)=k\left(E_{\text {elec }}+E_{a m p} * d^{2}\right)
$$

In which $E_{\text {elec }}$ indicates the power consumption of the electronic circuit and $E_{a m p}$ indicates the energy required to amplify the transmission signals to send a bit of data. In addition, the power consumption for the i-data receiver is measured by the following equation to receive a k-bit data packet.

$$
E_{r x}(i)=k * E_{\text {elec }}
$$

The energy consumption for nodes that are between the distance of the data sending node and the data receiving node will be equal to the sum of the data sending energy and the received data energy:

$$
E_{\text {cons }}(i)=\sum_{i=1}^{n}\left[E_{t x}(i)+E_{r x}(i)\right]
$$

The energy consumed by any type of node in this network, whether sender or receiver, can be calculated with the help of this equation.

Save the node address number

The node is stored in the database as a new route for sending when it is energetically acceptable. 

Networks (WSNs) Based on Software-Defined Network (SDN) Via Game of Theory

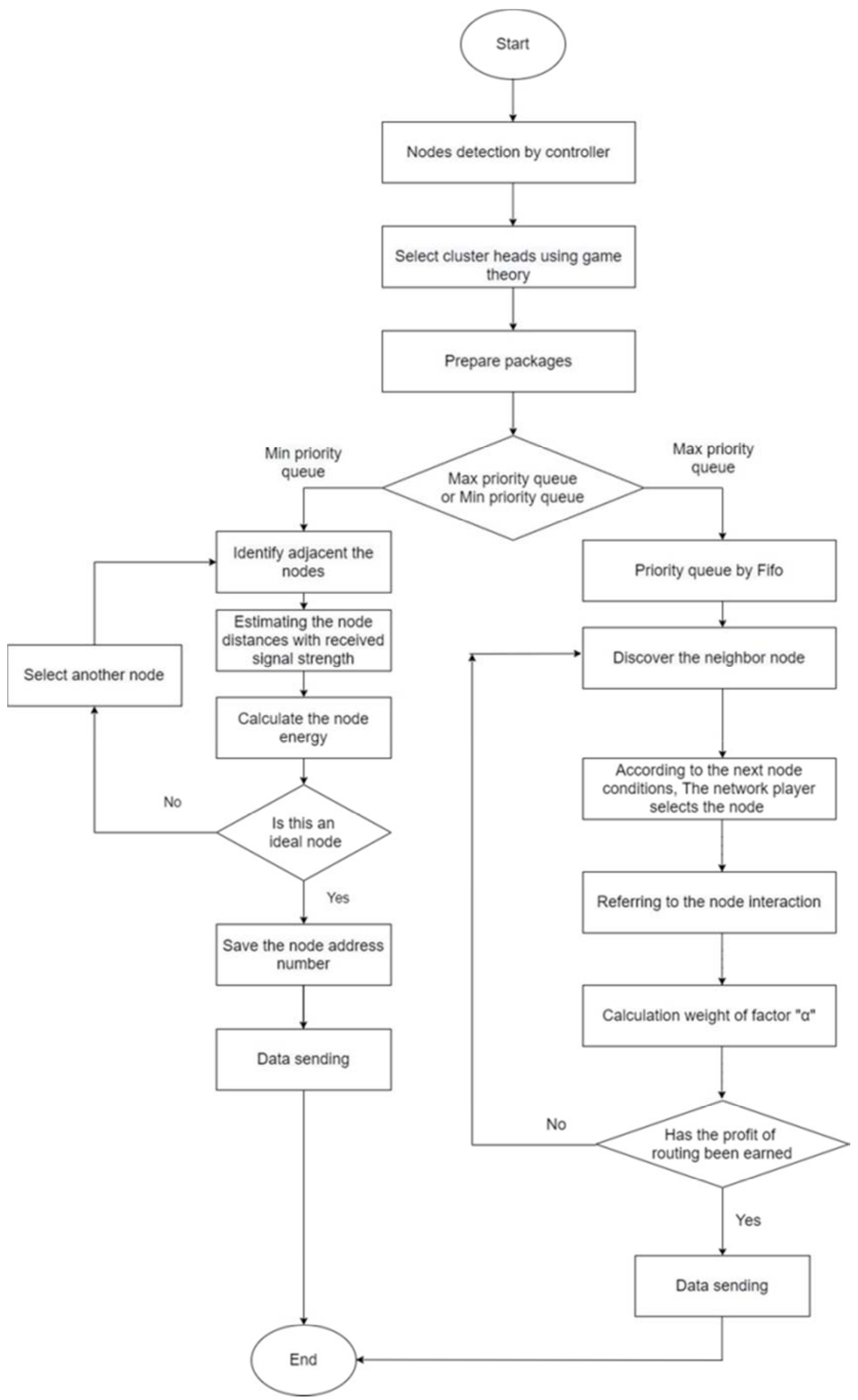

Figure 8. Flowchart of routing via Game theory. 


\section{Obtained Results}

The performance of the proposed method examined through Ant Colony Optimization algorithm (ACO), Genetic algorithm (GA), Particle Swarm Optimization algorithm (PSO), Firefly algorithm (FA), and Artificial Bee Colony algorithm $(\mathrm{ABC})$. The factors and results of the simulation are also examined.

\subsection{Simulation Environment}

NS3 is the most powerful software for simulating and analyzing computer and telecommunication networks. NS3 allows the user to simulate different aspects of physical layer, data link, and application layer. This software allows designers and researchers to simulate the performance of protocols, equipment, and network architectures with great accuracy. The predictions of this software are dramatically coincided with reality. The hardware used was an Intel Core i7 $3.2 \mathrm{GHz}$ processor with $16 \mathrm{~GB}$ of RAM. Windows 10 was also used.

\subsection{Simulation Parameters}

As shown in Table 4, the values used were network size, number of sinks, number of nodes, data packet size, initial energy of each node, buffer size of each node, and number of iterations were limited to facilitate the process. In addition in table 5 shown the scenario of this method.

Table 4. Simulation parameters.

\begin{tabular}{ll}
\hline Number of parameter & Value \\
\hline Number of base station & 1 \\
Number of nodes & 100 \\
Packet size & $256 \mathrm{~kb}$ \\
Initial energy of nodes & $0.5 \mathrm{j}$ \\
Radio range & $30 \mathrm{~m}$ \\
Number of repetitions & 200 \\
$\mathrm{E}_{\text {elec }}$ & $50 \mathrm{nj} / \mathrm{bit}$ \\
$\mathrm{E}_{\mathrm{amp}}$ & 0.0013 \\
$\mathrm{Pj}_{\mathrm{b}} \mathrm{bit} / \mathrm{m}^{4}$ & 10 \\
$\mathrm{E}_{\mathrm{fs}}$ & $10 \mathrm{pj} / \mathrm{bit} / \mathrm{m}^{4}$ \\
Normal reading range & $40-50$ \\
Threshold & 10 \\
\hline
\end{tabular}

Table 5. Used scenarios.

\begin{tabular}{lll}
\hline Number & Network size & Sink location \\
\hline 1 & $100 \mathrm{~m} \times 100 \mathrm{~m}$ & $50 \mathrm{~m}, 50 \mathrm{~m}$ \\
2 & $200 \mathrm{~m} \times 200 \mathrm{~m}$ & $200 \mathrm{~m}, 200 \mathrm{~m}$ \\
\hline
\end{tabular}

\subsection{Results}

In this section, the results of experiments are reviewed in NS3 software. In this section, the result of comparing the performance of different networks in the considered scenario were given in terms of the number of live network nodes, mean residual energy of nodes, total number of received packets in the sink, network life, total number of packets sent to the sink. It should be noted that the workload is the same for all cases in terms of performance. Game theory algorithm has obtained better answers to cluster selection and routing than metaheuristic algorithms. In Figure 9, it can be clearly seen that the live nodes of the proposed method are more live than the other methods. Subsequently, the $\mathrm{ABC}$ has obtained a more acceptable result, which is one of the most important parameters in the WSNs of node viability.

The number of packets received at the destination is divided by the total number of packages sent from the origin to calculate the package delivery rate at speed. The higher the ratio, the more packages have been successfully delivered to the destination given the total number of packages. In the proposed method, game theory has a higher package delivery rate than other methods because routing was assigned to data that wanted to send with high priority. If the data has higher priority, it sends the data to the $\mathrm{CH}$ by routing it closer. Figure 10 shows a comparison of the proposed methods in PDR.

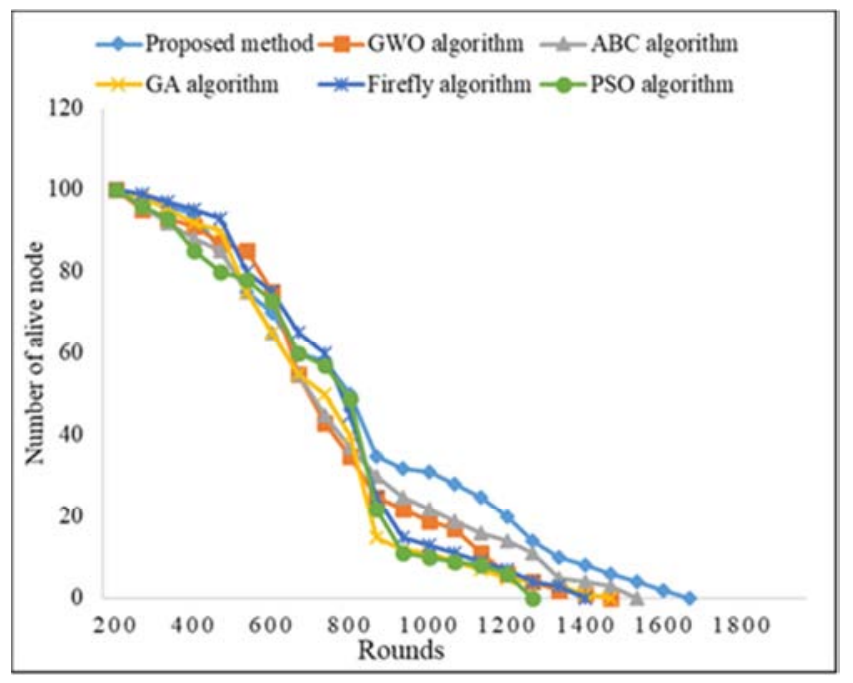

Figure 9. Comparison of a live nodes with other methods in first scenario.

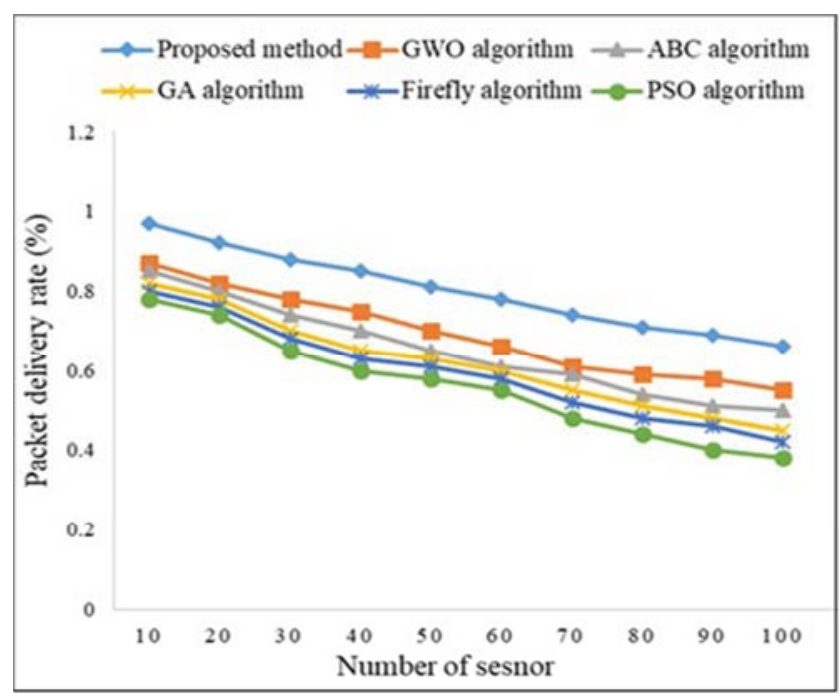

Figure 10. Comparison of package delivery rates with other methods in first scenario. 


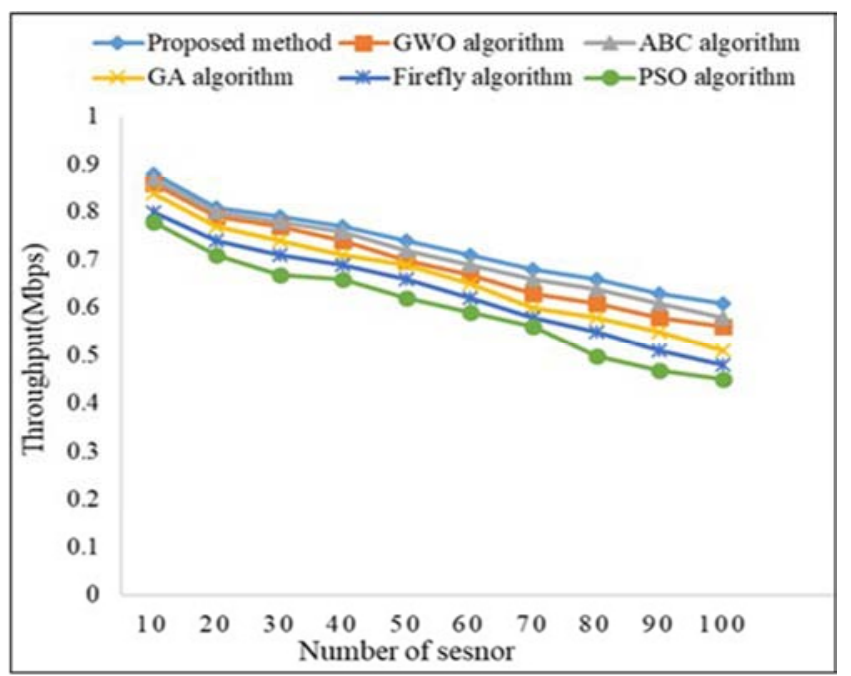

Figure 11. Throughput of nodes compared by other methods in first scenario.

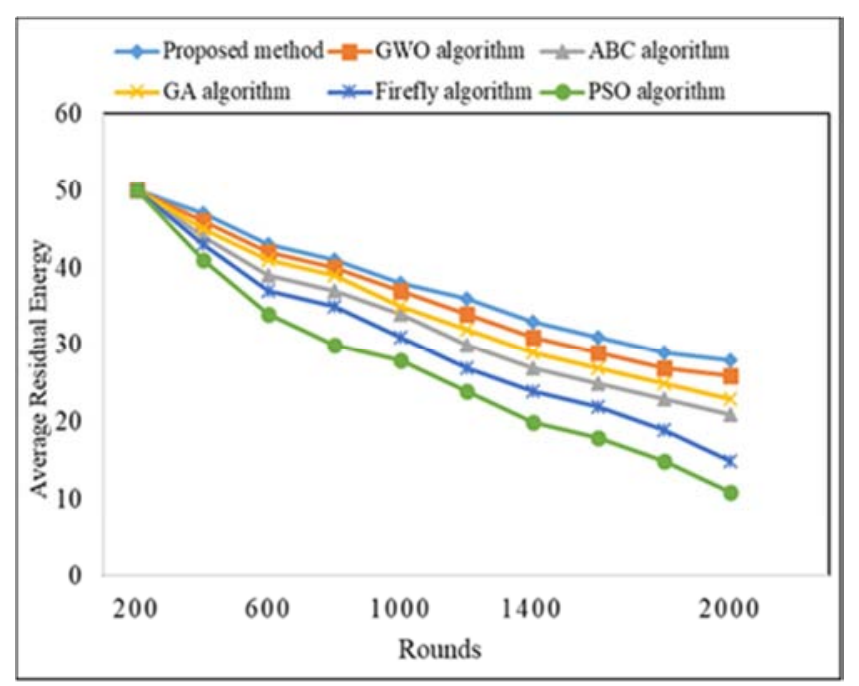

Figure 12. Residual energy of nodes compered by other methods in first scenario.

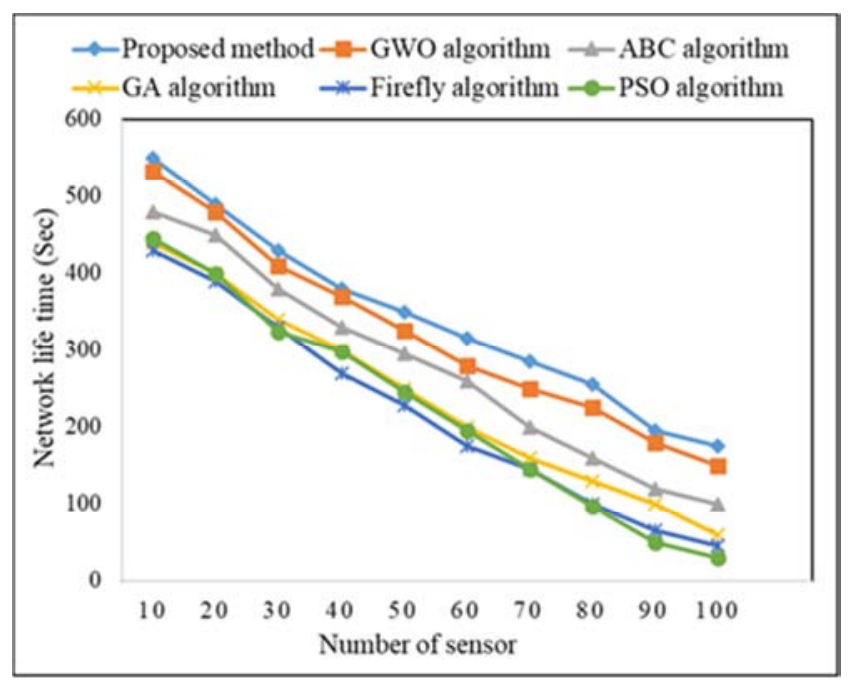

Figure 13. Comparison of network life time with other methods in first scenario.
Throughput refer to the actual number of packages delivered to the sink. This criterion indicates the ability of algorithms to transfer the desired number of packets to the sink. In the proposed method, a more acceptable result is obtained by comparing the load of the algorithms used. Figure 11 shows the throughput of nodes:

Residual energy of network nodes is the amount of energy available in network nodes to be used by nodes to transfer data to other nodes. According to Figure 12, it can be seen that the proposed method has the best conditions in terms of the mean residual energy of all network nodes.

Figure 13 compares the life time of different networks. Network lifetime means the time it takes for all nodes in a network to fail and the so-called network to shut down. According to the figure, it can be seen that the performance of the proposed method is better than the other discussed items.

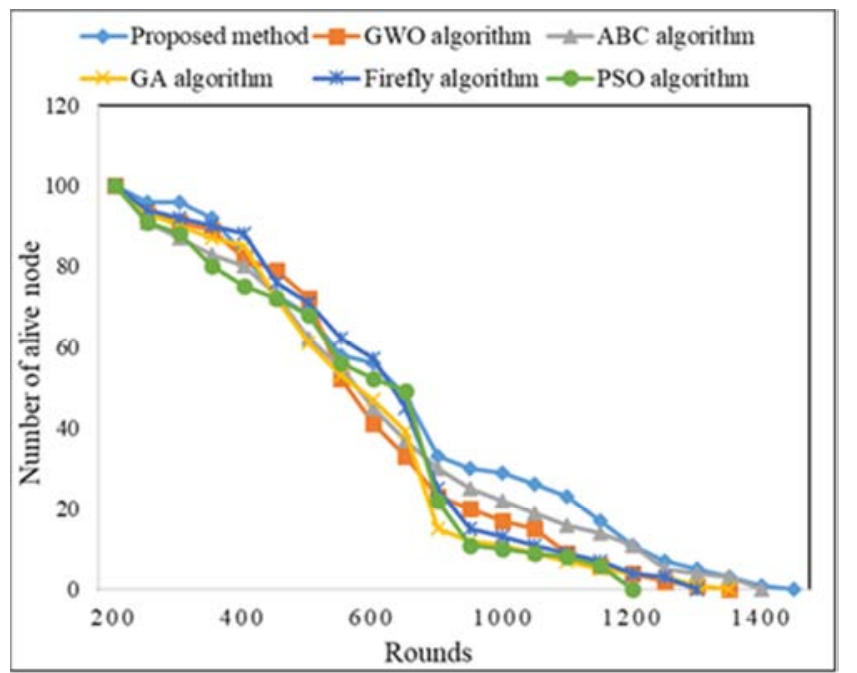

Figure 14. Comparison of a live node with other methods in second scenario.

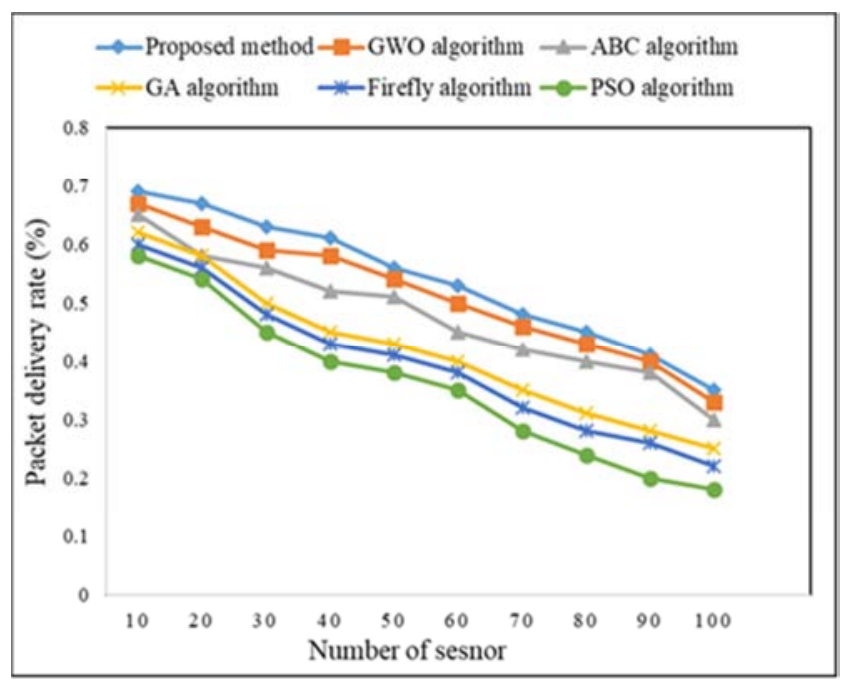

Figure 15. Comparison of a PDR with other methods in second scenario.

In the second scenario and figure 14 , the first level all method has approximately same, but after 600 rounds the 
PSO algorithm has the best answer compared with others, but suddenly this algorithm decrees sharply, in the live node the $\mathrm{ABC}$ algorithm and Game theory has a close competition but at the end, the proposed method has a good performance in compared with others.

In figure 15 , the proposed method has a good efficiency compared with others; also, in the second scenario, the network size has increased; furthermore, the proposed method has a good performance on a large scale. PSO algorithm again does not have good performance in these skills.

Throughput is a measure of total units of information a system can process in a given amount of time. In figure 16, the $\mathrm{ABC}$ and proposed method have good performance compared with another algorithm. FA and PSO algorithm in the 100 nodes have the same results.

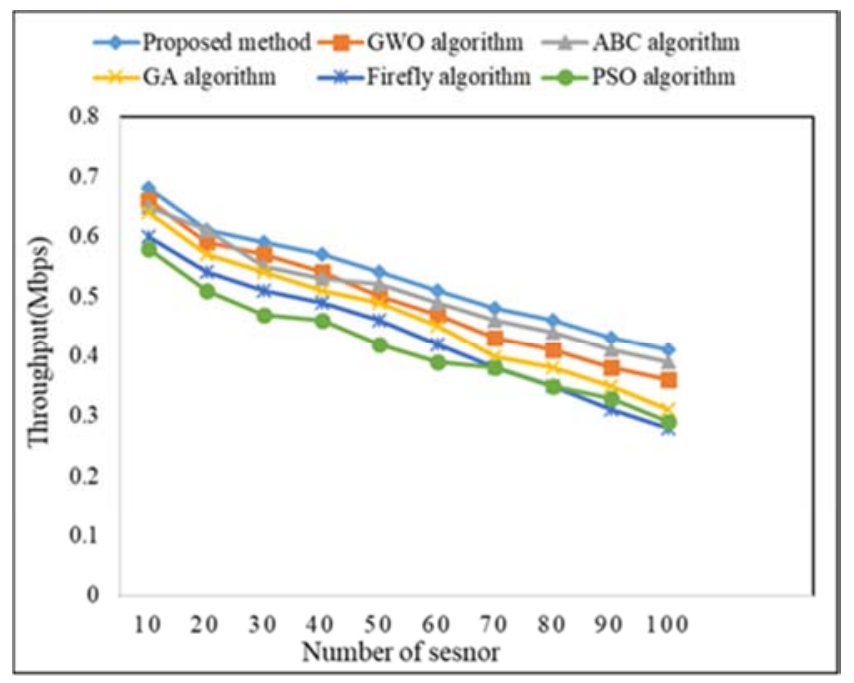

Figure 16. Comparison of a Throughput with other methods in second scenario.

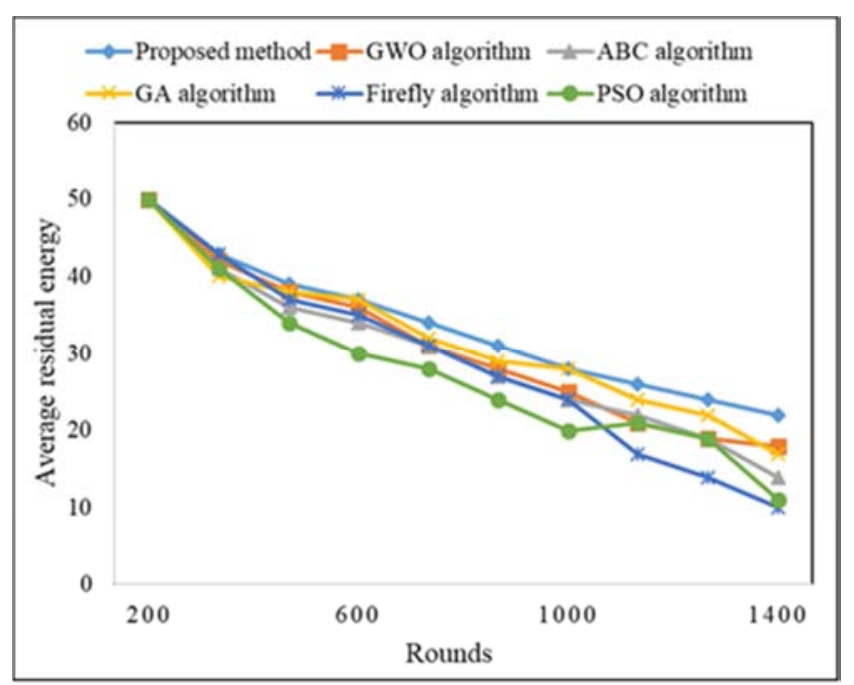

Figure 17. Comparison of average residual energy with other methods in the second scenario.

In Figure 17, all 6 methods are compared in terms of the average residual energy. As can be seen from the previous figure, the proposed method reduces energy consumption in the long time. In addition to maintaining the average energy, it is a reason for the energy level of nodes to be higher than other methods. Considering that, the issue of energy is the main challenge that has been solved in this research. Due to this method has the best performance among the three competitors in terms of the average energy of the nodes.

Lifetime of network is defined as the operational time of the network during which it is able to perform the dedicated task(s) and network lifetime is defined as the time until the first node exhausts its energy in the network. As expected from the proposed method in the second scenario, in the figure 18 , the proposed method has the best performance in terms of network life.

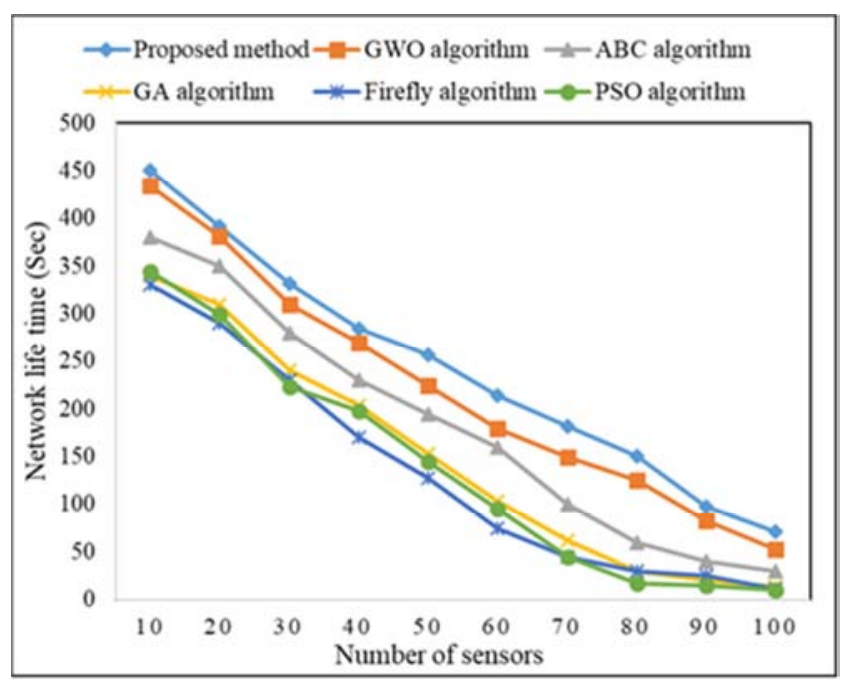

Figure 18. Comparison of network lifetime with other methods in the second scenario.

\section{Conclusions and Future Studies}

One of the most important problems of network experts regarding the establishment and maintenance of WSNs in various applications of these networks is the problem of battery limitation and the life of the nodes of this network. Since the efficiency of WSNs is directly correlated to the lifetime and maintenance of the network coverage of each of the nodes that make up these networks, so all surfaces of this type of network must be designed with energy awareness. Routing in the wireless sensor network is one the cases that energy awareness is a great help in increasing the life of the network. In this research, a new routing protocol and cluster selection are presented based on game theories. This routing and $\mathrm{CH}$ selection protocol sends information according to data priorities and also uses game theories for $\mathrm{CH}$ selection. The results of simulating the proposed algorithm and comparing it with the artificial bee algorithms, genetic algorithm, cuckoo algorithm, firefly algorithm, gray wolf algorithm showed acceptable results. 


\section{References}

[1] T. Jafarian, M. Masdari, A. Ghaffari, and K. Majidzadeh, "SADM-SDNC: security anomaly detection and mitigation in software-defined networking using C-support vector classification," Computing, vol. 103, no. 4, pp. 641-673, 2021.

[2] R. Engel, T. P. Barzilai, D. D. Kandlur, and A. Mehra, "Efficient classification, manipulation, and control of network transmissions by associating network flows with rule based functions," ed: Google Patents, 2003.

[3] B. A. A. Nunes, M. Mendonca, X.-N. Nguyen, K. Obraczka, and T. Turletti, "A survey of software-defined networking: Past, present, and future of programmable networks," IEEE Communications surveys \& tutorials, vol. 16, no. 3, pp. 16171634, 2014.

[4] H. Kim and N. Feamster, "Improving network management with software defined networking," IEEE Communications Magazine, vol. 51, no. 2, pp. 114-119, 2013.

[5] D. Kreutz, F. M. Ramos, P. E. Verissimo, C. E. Rothenberg, S. Azodolmolky, and S. Uhlig, "Software-defined networking: A comprehensive survey," Proceedings of the IEEE, vol. 103, no. 1, pp. 14-76, 2014.

[6] H. Kim, G. De Veciana, X. Yang, and M. Venkatachalam, "Distributed \$alpha \$-optimal user association and cell load balancing in wireless networks," IEEE/ACM Transactions on Networking, vol. 20, no. 1, pp. 177-190, 2011.

[7] S. A. Alabady, F. Al-Turjman, and S. Din, "A novel security model for cooperative virtual networks in the IoT era," International Journal of Parallel Programming, vol. 48, no. 2, pp. 280-295, 2020.

[8] S. S. Sefati and N. J. Navimipour, "A QoS-aware service composition mechanism in the Internet of things using a hidden Markov model-based optimization algorithm," IEEE Internet of Things Journal, pp. 1-1, 2021, doi: 10.1109/JIOT.2021.3074499.

[9] K. Maraiya, K. Kant, and N. Gupta, "Application based study on wireless sensor network," International Journal of Computer Applications, vol. 21, no. 8, pp. 9-15, 2011.

[10] M. Farooq-I-Azam, Q. Ni, and E. A. Ansari, "Intelligent energy efficient localization using variable range beacons in industrial wireless sensor networks," IEEE Transactions on Industrial Informatics, vol. 12, no. 6, pp. 2206-2216, 2016.

[11] A.-J. Garcia-Sanchez, F. Garcia-Sanchez, and J. Garcia-Haro, "Wireless sensor network deployment for integrating videosurveillance and data-monitoring in precision agriculture over distributed crops," Computers and electronics in agriculture, vol. 75, no. 2, pp. 288-303, 2011.

[12] M. T. Lazarescu and P. Poolad, "Asynchronous Resilient Wireless Sensor Network for Train Integrity Monitoring," IEEE Internet of Things Journal, vol. 8, no. 5, pp. 3939-3954, 2020.

[13] V.-P. Ha, T.-K. Dao, M.-H. Le, T.-H. Nguyen, and V.-T. Nguyen, "Design and Implementation of an Energy Simulation Platform for Wireless Sensor Networks," in 2020 International Conference on Multimedia Analysis and Pattern Recognition (MAPR), 2020: IEEE, pp. 1-6.
[14] O. Flauzac, C. Javier Gonzalez Santamaria, F. Nolot, and I. Woungang, "An SDN approach to route massive data flows of sensor networks," International Journal of Communication Systems, vol. 33, no. 7, p. e4309, 2020.

[15] A. Hawbani, X. Wang, L. Zhao, A. Al-Dubai, G. Min, and O. Busaileh, "Novel architecture and heuristic algorithms for software-defined wireless sensor networks," IEEE/ACM Transactions on Networking, vol. 28, no. 6, pp. 2809-2822, 2020.

[16] K. N. Qureshi, M. U. Bashir, J. Lloret, and A. Leon, "Optimized cluster-based dynamic energy-aware routing protocol for wireless sensor networks in agriculture precision," Journal of sensors, vol. 2020, 2020.

[17] V. Anand and S. Pandey, "New approach of GA-PSO - based clustering and routing in wireless sensor networks," International Journal of Communication Systems, vol. 33, no. 16, p. e4571, 2020.

[18] N. S. Khan, A. Hussain, M. Ali, A. Razzaq, and A. Ijaz, "Development of an Adaptive Energy Aware Routing Scheme for Wireless Sensor Networks."

[19] D. Kandris, A. Alexandridis, T. Dagiuklas, E. Panaousis, and D. D. Vergados, "Multiobjective Optimization Algorithms for Wireless Sensor Networks," ed: Hindawi, 2020.

[20] S. Dubey and C. Agrawal, "A survey of data collection techniques in wireless sensor network," International Journal of Advances in Engineering \& Technology, vol. 6, no. 4, p. 1664, 2013.

[21] D. Vinodha, E. M. Anita, and D. M. Geetha, "A novel multi functional multi parameter concealed cluster based data aggregation scheme for wireless sensor networks (NMFMPCDA)," Wireless Networks, vol. 27, no. 2, pp. 1111-1128, 2021.

[22] O. Younis, M. Krunz, and S. Ramasubramanian, "Node clustering in wireless sensor networks: Recent developments and deployment challenges," IEEE network, vol. 20, no. 3, pp. 20-25, 2006.

[23] V. Sundararaj, S. Muthukumar, and R. Kumar, "An optimal cluster formation based energy efficient dynamic scheduling hybrid MAC protocol for heavy traffic load in wireless sensor networks," Computers \& Security, vol. 77, pp. 277-288, 2018.

[24] C. Deepa and B. Latha, "HHSRP: a cluster based hybrid hierarchical secure routing protocol for wireless sensor networks," Cluster Computing, vol. 22, no. 5, pp. 10449-10465, 2019.

[25] V. Sharma, R. Agarwal, M. S. Gaur, V. Laxmi, and V. Vineetha, "ERA: an efficient routing algorithm for power, throughput and latency in network-on-chips," in IFIP International Conference on Network and Parallel Computing, 2010: Springer, pp. 481-490.

[26] B. D. Deebak and F. Al-Turjman, "A hybrid secure routing and monitoring mechanism in IoT-based wireless sensor networks," Ad Hoc Networks, vol. 97, p. 102022, 2020.

[27] P. L. R. Chze and K. S. Leong, "A secure multi-hop routing for IoT communication," in 2014 IEEE World forum on internet of things (WF-iot), 2014: IEEE, pp. 428-432.

[28] K. Guleria and A. K. Verma, "An energy efficient load balanced cluster-based routing using ant colony optimization for WSN," International Journal of Pervasive Computing and Communications, 2018. 
[29] W. A. Altakhayneh, M. Ismail, M. A. Altahrawi, and M. K. AbuFoul, "Cluster head selection using genetic algorithm in wireless network," in 2019 IEEE 14th Malaysia International Conference on Communication (MICC), 2019: IEEE, pp. 13-18.

[30] P. S. Rao, P. K. Jana, and H. Banka, "A particle swarm optimization based energy efficient cluster head selection algorithm for wireless sensor networks," Wireless networks, vol. 23, no. 7, pp. 2005-2020, 2017.

[31] M. Baskaran and C. Sadagopan, "Synchronous firefly algorithm for cluster head selection in WSN," The Scientific World Journal, vol. 2015, 2015.

[32] D. Karaboga, S. Okdem, and C. Ozturk, "Cluster based wireless sensor network routing using artificial bee colony algorithm," Wireless Networks, vol. 18, no. 7, pp. 847-860, 2012.

[33] M. Carlos-Mancilla, E. López-Mellado, and M. Siller, "Wireless sensor networks formation: approaches and techniques," Journal of Sensors, vol. 2016, 2016.

[34] F. Engmann, F. A. Katsriku, J.-D. Abdulai, K. S. Adu-Manu, and F. K. Banaseka, "Prolonging the lifetime of wireless sensor networks: a review of current techniques," Wireless Communications and Mobile Computing, vol. 2018, 2018.

[35] A. Pughat and V. Sharma, "A review on stochastic approach for dynamic power management in wireless sensor networks," Human-centric Computing and Information Sciences, vol. 5, no. 1, pp. 1-14, 2015.

[36] J. Zhou, H. Jiang, J. Wu, L. Wu, C. Zhu, and W. Li, "SDNbased application framework for wireless sensor and actor networks," IEEE Access, vol. 4, pp. 1583-1594, 2016.

[37] K. M. Modieginyane, B. B. Letswamotse, R. Malekian, and A. M. Abu-Mahfouz, "Software defined wireless sensor networks application opportunities for efficient network management: A survey," Computers \& Electrical Engineering, vol. 66, pp. 274-287, 2018

[38] Y. Duan, Y. Luo, W. Li, P. Pace, and G. Fortino, "Software defined wireless sensor networks: a review," in 2018 IEEE 22nd International Conference on Computer Supported Cooperative Work in Design ((CSCWD)), 2018: IEEE, pp. 826-831.
[39] Q. Xu and J. Zhao, "A WSN Architecture Based on SDN," 2016/12 2016, vol. Proceedings of the 4th International Conference on Information Systems and Computing Technology: Atlantis Press, pp. 159-163, doi: https://doi.org/10.2991/isct-16.2016.27. [Online]. Available: https://doi.org/10.2991/isct-16.2016.27

[40] S. Sefati, M. Abdi, and A. Ghaffari, "Cluster-based data transmission scheme in wireless sensor networks using black hole and ant colony algorithms," International Journal of Communication Systems, vol. 34, no. 9, p. e4768, 2021, doi: https://doi.org/10.1002/dac.4768.

[41] S. Sefati, M. Mousavinasab, and R. Zareh Farkhady, "Load balancing in cloud computing environment using the Grey wolf optimization algorithm based on the reliability: performance evaluation," The Journal of Supercomputing, 2021/05/19 2021, doi: 10.1007/s11227-021-03810-8.

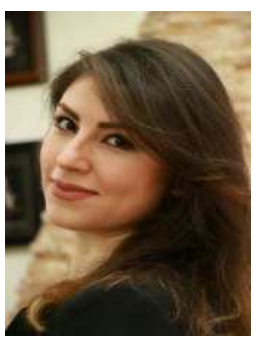

Sara Ghiasi Tabrizi is graduated M.Sc. of information technology in [2015]-[2017] in Islamic Azad University her thesis was Data storage based on genetic algorithm in wireless sensor networks to reduce access time. She is researching about Internet of things, Cloud computing and Wireless sensor networks. Sara's skills in web programming, C\# programming language, Bootstrap and access and SQL server databases. She also has 6 years' experience of security solutions and time attendance services.

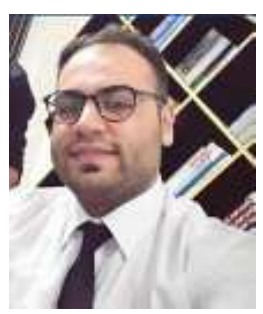

Seyed Salar Sefati received his M.Sc. degree in computer engineering-software from Tabriz branch, IAU (Islamic Azad University), in 2020. He is in the top 5 students among the 136 master students. He is the assistant lecture in Institute of Higher Education Roshdiyeh, Tabriz Iran. His research interests are mainly in the field of Wireless Sensor Networks (WSNs), Vehicular Ad Hoc Networks (VANETs), Internet of things (IoT), and Cloud Computing. 